\title{
Análisis bibliométrico de la incontinencia urinaria masculina. Una mirada de los últimos 20 años
}

\section{Bibliometric Analysis of Urinary Incontinence. An Overview of the Last 20 Years}

\author{
Catalina Sánchez Basto ${ }^{10}$ Adriana Chaves Parra ${ }^{2}$ Catalina Osorio Ospino ${ }^{3(1)}$ \\ María Paula Saenz Becerra ${ }^{4}$ Johanna Vega ${ }^{5(0)}$ Daisy Ximena Saavedra ${ }^{6(1)}$ Melanie López de Mesa ${ }^{7(0)}$ \\ Irma A. Ospina-Galeano ${ }^{8(1)}$ Catalina Solano Mendoza9 9
}

${ }^{1}$ Clínica Nueva El Lago, Bogotá, Colombia

2 Uronorte, Cúcuta, Norte de Santander, Colombia

${ }^{3}$ Clínica Comfamiliar Risaralda, Risaralda, Colombia

${ }^{4}$ Clínica Universidad de La Sabana, Cundinamarca, Colombia

${ }^{5}$ Hospital de la Samaritana, Cundinamarca, Colombia

${ }^{6}$ Cooperativa Especializada de Servicios Urológicos del Tolima (Urotol), Ibagué, Tolima, Colombia

${ }^{7}$ Hospital San José, Bogotá, Colombia

${ }^{8}$ Hospital Universitario San Vicente Fundación, Medellín, Antioquia,

Colombia

${ }^{9}$ Serivicio de Urología, Uroclin, Medellín, Colombia

Urol Colomb 2021;30(4):e319-e326.
Dirección para correspondencia Catalina Sánchez Basto, MD, calle 131 A \# 19-30 casa. La calleja, Bogotá, Colombia, Cel: 3166171514 (e-mail: cata_sanchez27@hotmail.com).

\section{Resúmen}

\section{Palabras Clave}

- incontinencia urinaria

- análisis bibliométrico

- bibliométrico

- base de datos Web of Science

- análisis de visualización

- análisis de citaciones
Introducción y Objetivo En las últimas décadas, la incidencia y prevalencia de la incontinencia urinaria han venido en aumento. Existen numerosas publicaciones sobre este tema, se han introducido nuevos dispositivos, y se han modificado las líneas de manejo de esta patología. El objetivo de este estudio es realizar un análisis bibliométrico de las publicaciones sobre incontinencia urinaria masculina en las revistas indexadas.

Métodos Se realizó un análisis bibliométrico descriptivo de corte retrospectivo de la literatura médica disponible en la base de datos Web of Science (WOS) en relación con incontinencia urinaria masculina entre 2002 y 2020. Por medio de la WOS, se obtuvieron las variables a analizar, y se elaboraron tablas y gráficas en el programa Microsoft Office Excel 2017.

Resultados Encontramos un total de 498 publicaciones en 88 revistas. Hubo un crecimiento exponencial de las publicaciones a partir de 2006. Las 3 revistas con mayor porcentaje de publicaciones fueron: J Urol, con 25,2\% (125), Neurol Urodyn, con 16,5\% (82), European Urology Journal, con 11,1\% (55); sólo 5 de las 20 revistas con más publicaciones tuvieron un factor de impacto $\geq 3$. El país que tuvo mayor porcentaje fue Estados Unidos con 21,2\% (105), seguido por países europeos. En América Latina, el país con mayor número de publicaciones fue Brasil, con 2,6\% (13). No se encontraron estudios colombianos con esta búsqueda específica. recibido

26 de octubre de 2020

aceptado

02 de mes de julio de 2021
DOI https://doi.org/ 10.1055/s-0041-1740268. ISSN 0120-789X. e ISSN 2027-0119.
(C) 2021. Sociedad Colombiana de Urología. All rights reserved. This is an open access article published by Thieme under the terms of the Creative Commons Attribution-NonDerivative-NonCommercial-License, permitting copying and reproduction so long as the original work is given appropriate credit. Contents may not be used for commercial purposes, or adapted, remixed, transformed or built upon. (https://creativecommons.org/ licenses/by-nc-nd/4.0/)

Thieme Revinter Publicações Ltda., Rua do Matoso 170, Rio de Janeiro, RJ, CEP 20270-135, Brazil 


\section{Abstract}

\section{Keywords}

- urinary incontinence

- bibliometric analysis

- bibliometric

- Web of Science database

- visualization analysis

- citation analysis
Conclusión Este es el primer estudio bibliométrico sobre incontinencia urinaria masculina. Con el tiempo, han aumentado las publicaciones acerca de este tema, y la mayoría está concentrada en revistas de Estados Unidos y Europa. No es predominante la publicación en revistas de alto factor de impacto, y es muy baja la cantidad de publicaciones que aporta Colombia y los demás países latinoamericanos. Introduction In the last decades, the incidence and prevalence of urinary incontinence have been increasing. There are many publications about this subject, there are new devices, and the treatment of this condition has changed with time. The objective of the present study was to perform a bibliometric analysis of the publications about male urinary incontinence in the indexed journals.

Methods We performed a retrospective descriptive bibliometric analysis of the medical literature available on the Web of Science (WOS) database about male urinary incontinence between 2002 and 2020. Through the WOS database, the variables to be analyzed were obtained, and tables and graphs were created using the Microsoft Office Excel 2017.

Results We found 498 publications in 88 journals. Since 2006, there has been an exponential growth in publications. The 3 journals with the highest percentage of publications were: J Urol, with $25.1 \%$ (125), Neurol Urodyn, with $16.5 \%$ (82), European Urology Journal, with $11.4 \%$ (57); only 5 of the 20 journals with the highest number of publications had an impact factor $\geq 3$. The country with the highest percentage of publications was the United States with 21,2\% (105), followed by European countries. In Latin America, the country with the highest number of publications was Brazil, with 2,6\% (13). No Colombian studies were found with this specific research.

Conclusion The present is the first bibliometric analysis on male urinary incontinence. Over time, publications on this topic have increased, and most of them are concentrated in journals from the United States and Europe. The publication in journals with a high impact factor is not predominant, and the number of publications from Colombia and other Latin American countries is very low.

\section{Introducción}

Según la International Continence Society (ICS), la incontinencia urinaria es un síntoma de la fase de llenado de la vejiga, y se refiere a cualquier perdida involuntaria de orina. En Norte América, se ha reportado una prevalencia entre el $5.3 \%-45.8 \%{ }^{3}$ Esta prevalencia aumenta con la edad. ${ }^{1,2}$ En los hombres, la incontinencia urinaria de urgencia es la presentación más común, seguida por la incontinencia urinaria mixta, y, por último, la incontinencia urinaria de esfuerzo. ${ }^{2-4}$

La incontinencia posterior a prostatectomía radical persiste en aumento pese al perfeccionamiento de la técnica quirúrgica, y esto se debe al aumento del número de procedimientos que se realizan. ${ }^{5}$ Existen numerosas series que reportan los desenlaces con las diferentes intervenciones quirúrgicas para incontinencia urinaria postprostatectomía; sin embargo, en una revisión reciente resaltan la necesidad de contar con estudios clínicos prospectivos. $^{6}$

La incontinencia urinaria es un síntoma muy molesto que generalmente está asociado con ansiedad, depresión y disminución de la calidad de vida, lo que significa una carga económica importante para los sistemas de salud. ${ }^{7}$ Además, está asociada a comorbilidades como afección cognitiva, infartos, infecciones urinarias, patología prostática, y diabetes mellitus. ${ }^{4}$ Se ha demostrado que su efecto negativo en la calidad de vida tiene relación con otras afecciones relacionadas con síntomas gastrointestinales y problemas psiquiátricos. ${ }^{7}$

El manejo de esta condición depende de su causa, fisiopatología, nivel de afección de calidad de vida, comorbilidades, y contexto del paciente. La nueva generación de dispositivos ha cambiado el abordaje terapéutico de esta patología. ${ }^{8}$ Por estos motivos, es clave tener suficiente evidencia científica para determinar un manejo basado en la evidencia.

Teniendo en cuenta la gran cantidad de publicaciones de incontinencia urinaria y los distintos tipos de estudio, impacto científico, y las distintas revistas en las que estos son publicados, decidimos hacer un análisis de estas publicaciones para caracterizarlas y describir su comportamiento en el tiempo. Esta caracterización permite identificar la calidad de las publicaciones, tomar decisiones clínicas con más información, descubrir las falencias que persisten en las publicaciones acerca de 
incontinencia urinaria masculina, y posiblemente abrir la puerta a nuevos estudios en nuestro medio.

Por esas razones, el objetivo de este estudio fue realizar un análisis bibliométrico de la evidencia científica publicada acerca de incontinencia urinaria masculina en los últimos veinte años.

\section{Métodos}

Se realizó un análisis bibliométrico descriptivo, de corte retrospectivo, de la literatura médica disponible en la base de datos Web of Science (WOS), en relación con incontinencia urinaria masculina, desde el año 2002 hasta el año 2020.

\section{Búsqueda}

La WOS fue consultada en julio de 2020 utilizando la siguiente estrategia de búsqueda: (male urinary incontinence) [Title/Abstract]. Se incluyeron artículos originales de investigación, artículos de revista, ensayos, informes científicos, y reseñas, con el fin de analizar toda la literatura médica indexada en relación con este tema.

\section{Análisis de Bibliometría}

El análisis estadístico se realizó a través de la herramienta estadística de la WOS para la obtención de la información de las variables a analizar (número total de publicaciones $\mathrm{y}$ revistas, número y porcentaje de publicaciones por revista y país, patrón de producción científica a lo largo del período estudiado, autores, áreas de investigación, tipo de documentos, artículos más citados). Para la obtención del factor de impacto (FI), utilizamos la herramienta Journal Citation Reports 2017/2018 (Clarivate, Londres, RU) de las revistas encontradas para estimar la calidad de cada una de estas. $^{9}$

\section{Análisis por Mapeo}

Se realizó un mapeo bibliográfico utilizando el programa VOSViewer (Centre for Science and Technology Studies, Universiteit Leiden, Leiden, Países Bajos), el cual permite identificar las áreas de investigación más importantes sobre el tema y las características en la tendencia de palabras clave. Con el mismo programa, se evaluaron las correlaciónes de palabras clave y de palabras clave en relación con título y resumen, la cual se realizó mediante técnicas de agrupamiento $^{10,11}$ explorando las interacciones de palabras clave de título y resumen de la literatura encontrada, con el fin de mapear el dominio del conocimiento científico y revelarle al lector una manera simple y rápida de ver los datos. Se adoptó el análisis de coocurrencia para investigar las áreas populares y direcciones de investigación, y fue clave para monitorear desarrollos en áreas científicas.

\section{Resultados}

\section{Análisis de Bibliometría}

Se encontró un total de 498 publicaciones en 88 revistas desde 2002 a 2020 - Figura 1. A partir del 2006, se inició un crecimiento exponencial, obteniendo el 89,4\% (445) del total de las referencias encontradas en la WOS.

En total, se encontraron 88 revistas. El 92.1\% (459) de las publicaciones se encuentran en las 20 revistas mencionadas en la - Tabla 1, así como el número de artículos publicados en cada una de estas y el FI para el año 2018 según el Journal Citation Reports. Las 5 revistas con mayor porcentaje de publicaciones fueron: J Urol, con 25.2\% (125), Neurol Urodyn, con 16,5\% (82), European Urology Journal, con 11,4\% (57), Progress Urologe, con 11\% (55), y BJU Int, con 8,2\% (41), como se ve en la - Tabla 1. Sólo 5 de las 20 revistas con más publicaciones tuvieron un $\mathrm{FI} \geq 3$.

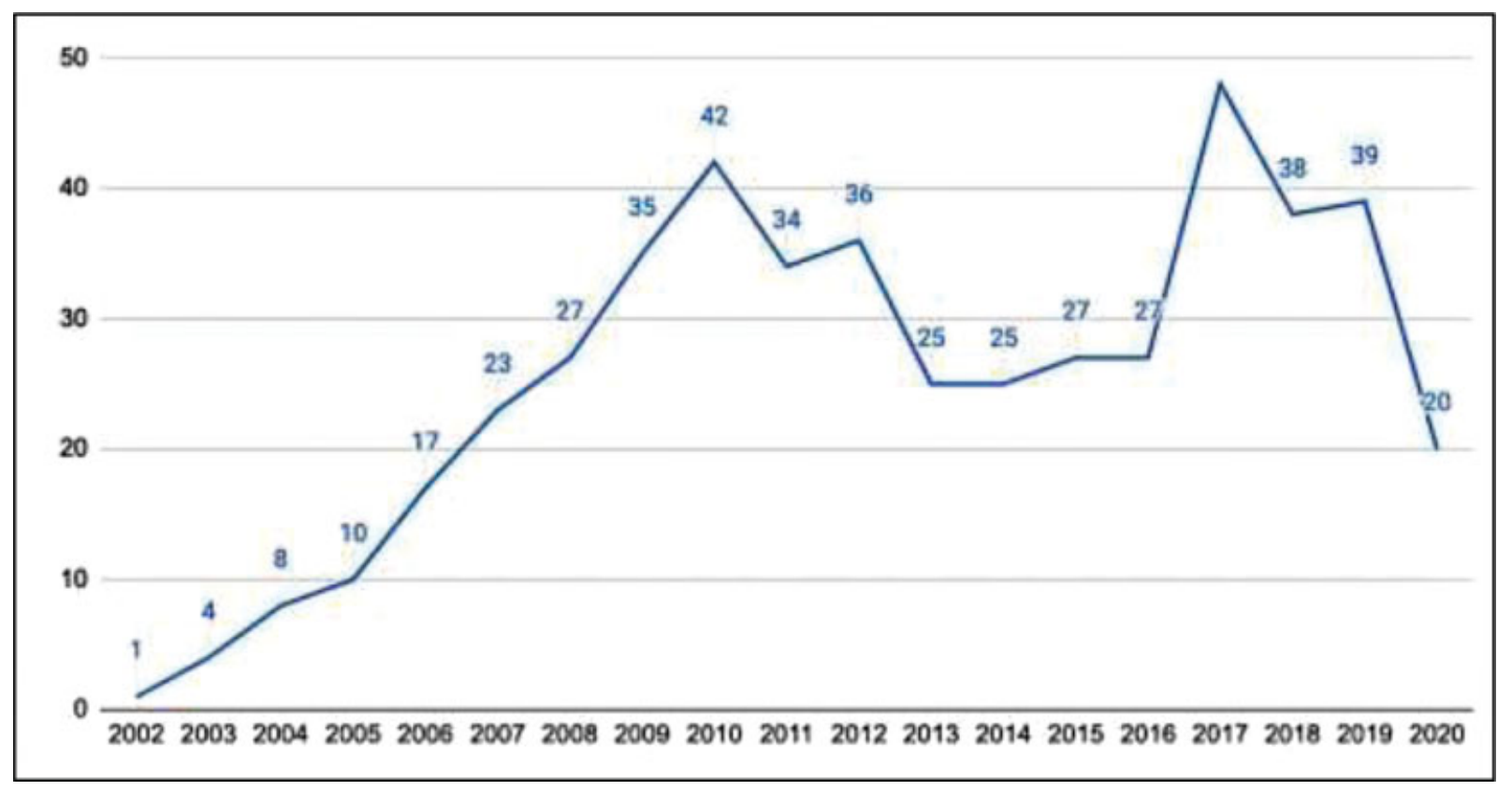

Fig. 1 Tendencia de publicaciones acerca de incontinencia urinaria masculina por año. 
Tabla 1 Listado de revistas con artículos sobre incontinencia urinaria masculina de 2002 a 2020, su factor de impacto para 20172018, el número de artículos, el idioma, y el país

\begin{tabular}{|l|l|l|l|l|}
\hline Revista & Artículos & Factor de impacto (2017-2018) & País & Idioma \\
\hline Journal of Urology & 125 & 5.64 & Estados Unidos & Inglés \\
\hline Neurourology and Urodynamics & 82 & 2.36 & Estados Unidos & Inglés \\
\hline European Urology & 57 & 17.29 & Países Bajos & Inglés \\
\hline Progres en Urologie & 55 & 0.47 & Francia & Francés \\
\hline BJU Int & 41 & 4.52 & Inglaterra & Inglés \\
\hline Urology & 28 & 1.86 & Estados Unidos & Inglés \\
\hline Int Journal of Urology & 20 & 2.1 & Japón & Inglés \\
\hline Journal of Sexual Medicine & 16 & 3.54 & Inglaterra & Inglés \\
\hline Der Urologe Ausg & 12 & $*$ & Alemania & Inglés \\
\hline Urologe & 12 & 0.49 & Alemania & Alemán \\
\hline Lower Urinary Tract Symptoms & 12 & 1.2 & Japón & Inglés \\
\hline Int Braz J Urol & 10 & 1.34 & Brasil & Inglés \\
\hline Journal of Endourology & 7 & 2.32 & Estados Unidos & Inglés \\
\hline Urologia Internationalis & 7 & 1.69 & Suiza & Inglés \\
\hline Actas Urológicas Españolas & 6 & 1.13 & España & Español \\
\hline Current Opinion in Urology & 6 & 2.05 & Inglaterra & Inglés \\
\hline International Neurourology Journal & 5 & 1.79 & Corea del Sur & Inglés \\
\hline World Journal of Urology & 5 & 3.21 & Estados Unidos & Inglés \\
\hline International Urology and Nephrology & 4 & 1.84 & Países Bajos & Inglés \\
\hline Pelvi Perineologie & 3 & $*$ & Francia & Francés \\
\hline
\end{tabular}

Nota: *No presenta Fl para 2017-2018.

La producción total de publicaciones se originó en 44 países, y el $75,7 \%$ se publicó en los 10 primeros países listados en la - Figura 2. De estos, el que tiene mayor porcentaje es Estados Unidos, con 21\% (105), seguido por Francia, con $10,4 \%$ (52), Alemania, con 9,8\%(49), e Italia, con 8,8\% (44). El país de América Latina con mayor número de publicaciones es Brasil, con 2,6\% (13), seguido de Argentina, con $0.8 \%$ (4).

\section{Áreas de Investigación y Tipos de Documento}

Dentro de las áreas de investigación, encontramos que el gran porcentaje está distribuido entre nefrología, urología, y cirugía (96\%); sin embargo, existe una gran variedad en áreas de cooperación, como se grafica en la - Figura 3a. Dentro del tipo de publicación, se distribuyen en su mayoría entre resúmenes de congresos y artículos, siguiéndole en proporción editoriales y revisiones (-Figura 3b).

\section{Artículos Más Citados}

En la - Tabla 2, se exponen los diez artículos más citados. El que ocupa el primer puesto, con 221 citaciones, es: "Transobturator sling suspension for male urinary incontinence including post-radical prostatectomy", de Rehder et al., publicado en el 2007 en la revista European Urology Journal, la cual tiene un FI alto (17,29). El 70\% tratan de dispositivos y manejo quirúrgico.

\section{Análisis de Mapeo}

Los 498 artículos se visualizaron utilizando el VOSviewer para identificar las áreas de investigación más importantes en incontinencia urinaria masculina. Las palabras clave (repetidas más de cinco veces) utilizadas en títulos y resúmenes fueron analizadas para investigar cómo los conceptos y los temas han evolucionado.

La - Figura 4a presenta las 226 palabras clave divididas en 7 grupos. A continuación, se describen los tres grupos principales (mayoría de ítems). Grupo 1 (rojo) "manejo quirúrgico": incontinencia urinaria de esfuerzo severa, esfínter, esfínter urinario artificial, cabestrillo ajustable; grupo 2 (verde) "tipo incontinencia": mecanismos, síntomas, prevalencia, impacto; grupo 3 (azul) "resultados": respuesta clínica, falla en el tratamiento, tasa de curación. En el mapeo en línea de tiempo (- Figura 4b), vemos que se han venido agregando a la línea de investigación en los últimos años temas como: robótica, atoms, y dispositivos ajustables.

\section{Discusión}

La incontinencia urinaria afecta negativamente la calidad de vida, aumenta el riesgo de institucionalización, y empeora el estado funcional de los pacientes; en un estudio ${ }^{12}$ con más de dos mil pacientes, se demostró que la incontinencia aumenta 


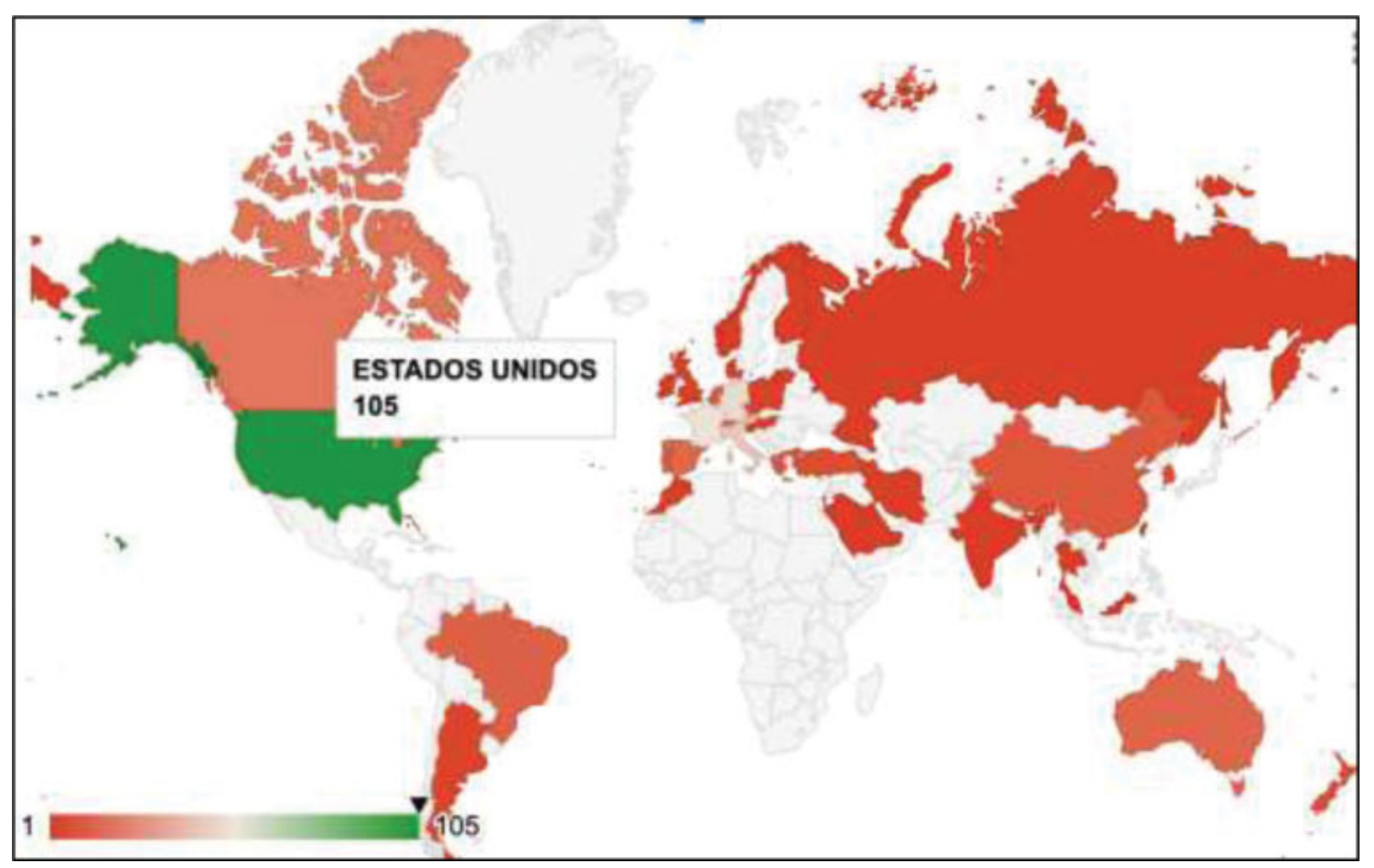

Fig. 2 Distribución de países con publicaciones acerca incontinencia urinaria masculina de 2002 a 2020.
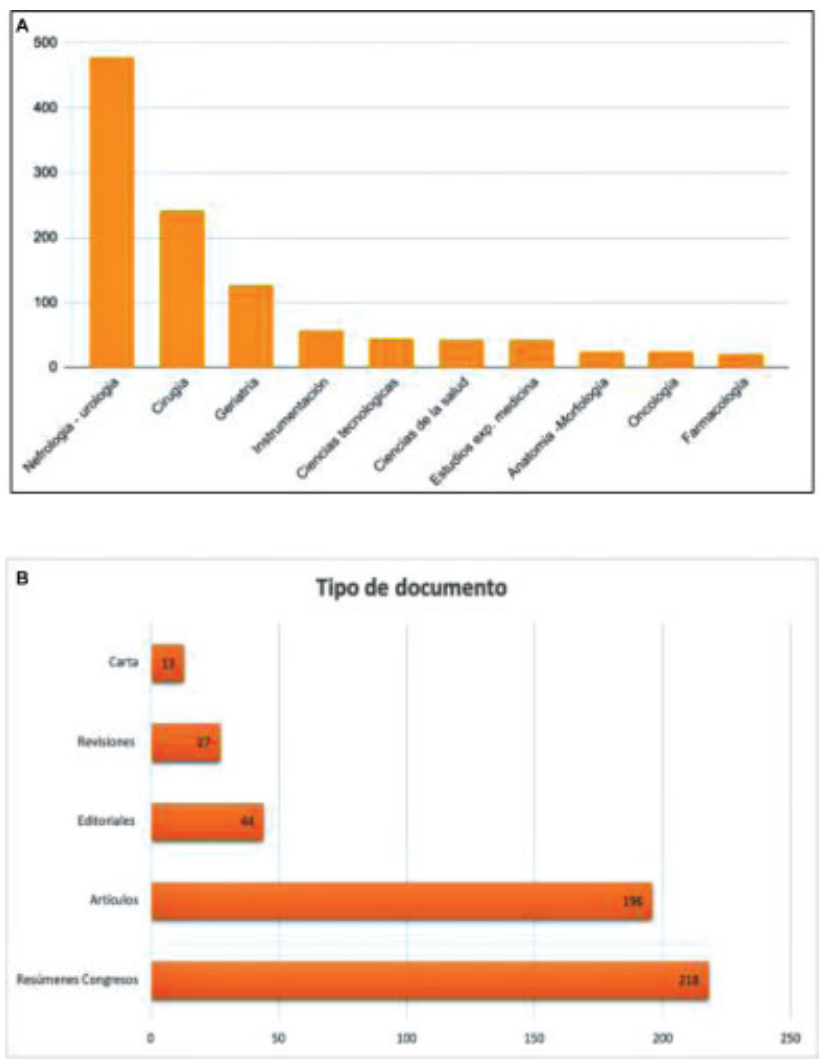

Fig. 3 Gráfica de áreas de investigación y tipo de publicación. (A) Áreas de investigación. (B). Tipos de documento. los índices de fragilidad; ${ }^{12} \mathrm{y}$, en otro estudio, ${ }^{13}$ se demostró que aumenta el riesgo de depresión. Por consiguiente, es importante conocer el patrón y tendencias bibliométricas de las publicaciones sobre esta condición, dado que esto afecta los esquemas de manejo y las publicaciones futuras.

En nuestro estudio, encontramos que, a partir de 2006, hubo un crecimiento exponencial de las publicaciones, con un $89,4 \%$ desde ese entonces; esto puede explicarse por la popularidad que adquiere la técnica de prostatectomía radical publicada por Walsh en 1988, y por el aumento en la incidencia de incontinencia urinaria masculina, seguido de la introducción de nuevos dispositivos para corregir la incontinencia en los últimos veinte años. ${ }^{6,14}$ La mayoría de los artículos son específicos de tratamiento quirúrgico (técnicas, materiales y desenlaces). Este hallazgo se observa en la publicación de Liu et al. ${ }^{15}$ acerca de dispositivos anti-incontinencia; los autores reportan que los procedimientos con cintas en hombres aumentaron de $32,7 \%$ en 2004 a 45,5\% en 2013.

Por otro lado, al analizar la producción por países, predominaron las publicaciones en Estados Unidos y Europa, lo cual concuerda con lo encontrado en un análisis bibliométrico de vejiga neurogénica de $2015,{ }^{16}$ en el cual los autores reportan que Estados Unidos fue el país con mayor número de publicaciones, resaltan que sus mayores competidores en producción son Europa occidental y China, y sugieren que este fenómeno se debe probablemente a los incentivos gubernamentales para la 


\begin{tabular}{|c|c|c|c|c|c|c|c|c|c|c|}
\hline 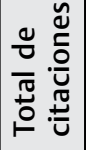 & $\overline{\mathbb{N}}$ & 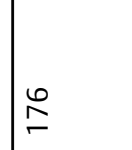 & $\stackrel{m}{m}$ & 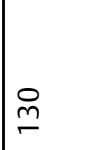 & $\underset{⿱}{\check{I}}$ & ¿ & $\stackrel{m}{o}$ & 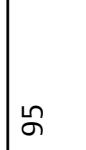 & ৪ & $\stackrel{\infty}{\wedge}$ \\
\hline 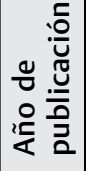 & 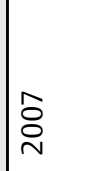 & 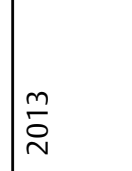 & $\frac{\sim}{\grave{N}}$ & 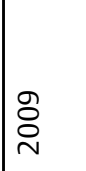 & 㝑 & 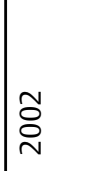 & ஓे & 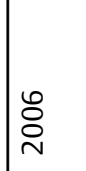 & $\overline{\tilde{N}}$ & 을 \\
\hline 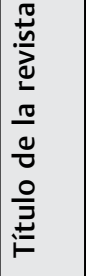 & 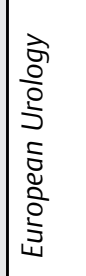 & 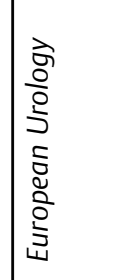 & 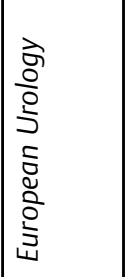 & 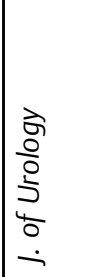 & 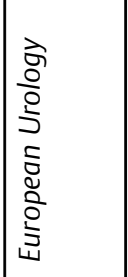 & $\begin{array}{l}\text { के } \\
\frac{0}{0} \\
\frac{0}{2} \\
4 \\
\\
-1\end{array}$ & 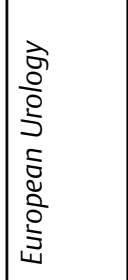 & 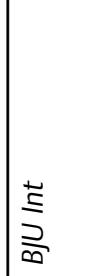 & $\mid \frac{\vec{\Sigma}}{\supset}$ & 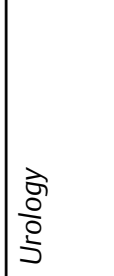 \\
\hline 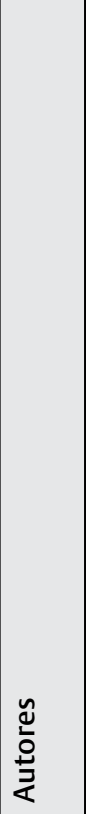 & 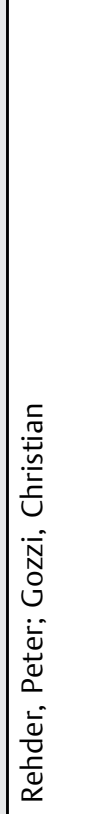 & 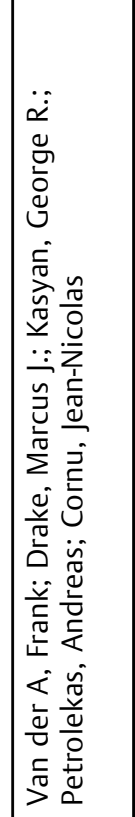 & 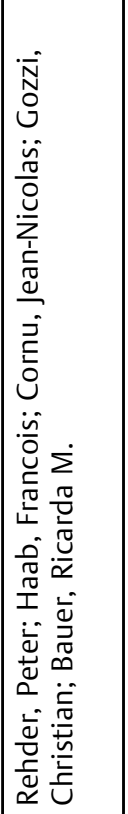 & 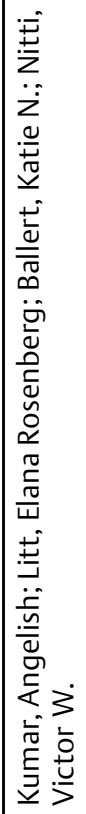 & 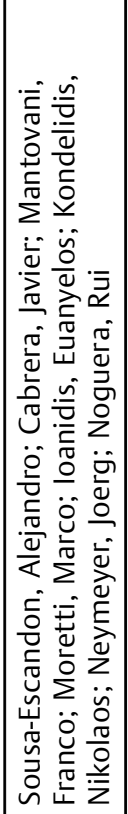 & 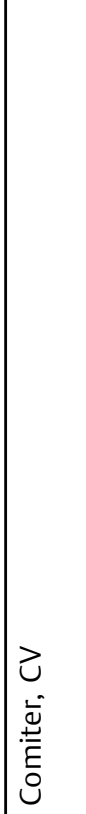 & 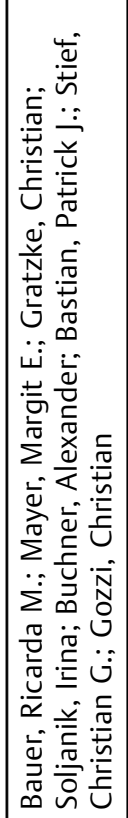 & 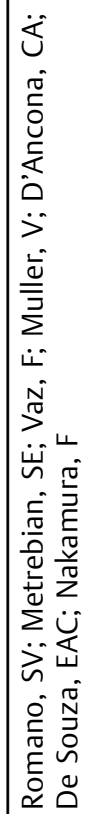 & 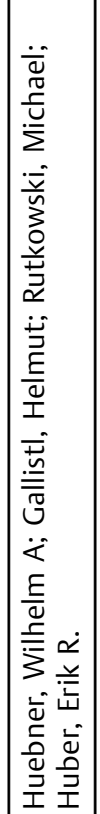 & 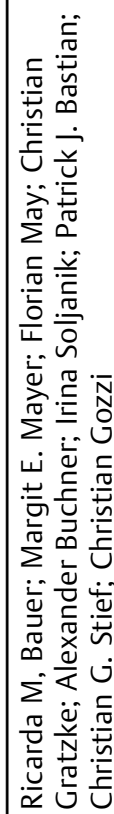 \\
\hline$\stackrel{\circ}{\stackrel{\circ}{E}}$ & 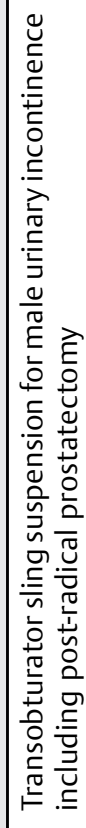 & 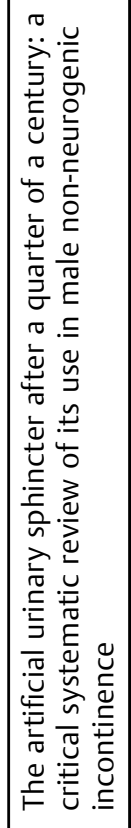 & 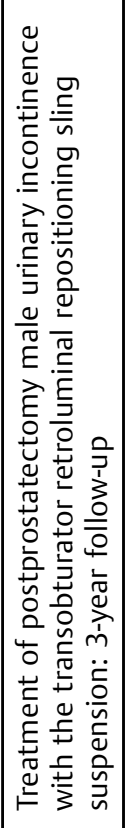 & 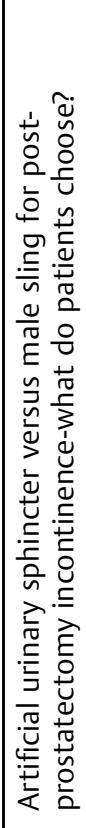 & 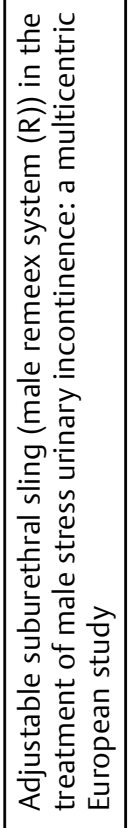 & 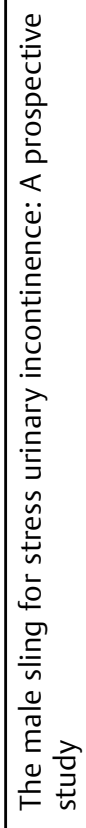 & 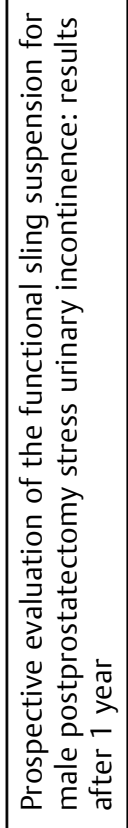 & 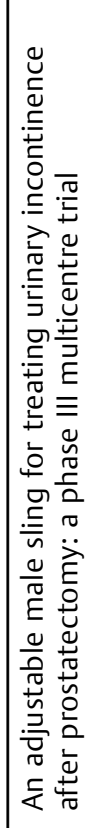 & 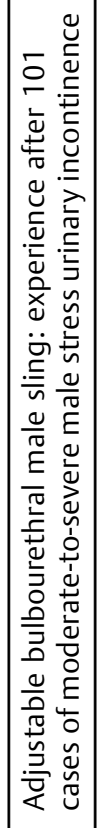 & 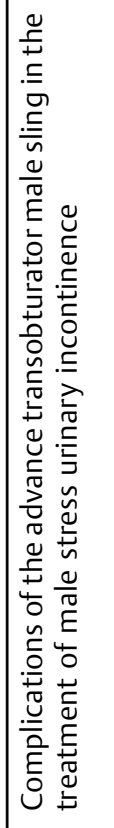 \\
\hline
\end{tabular}



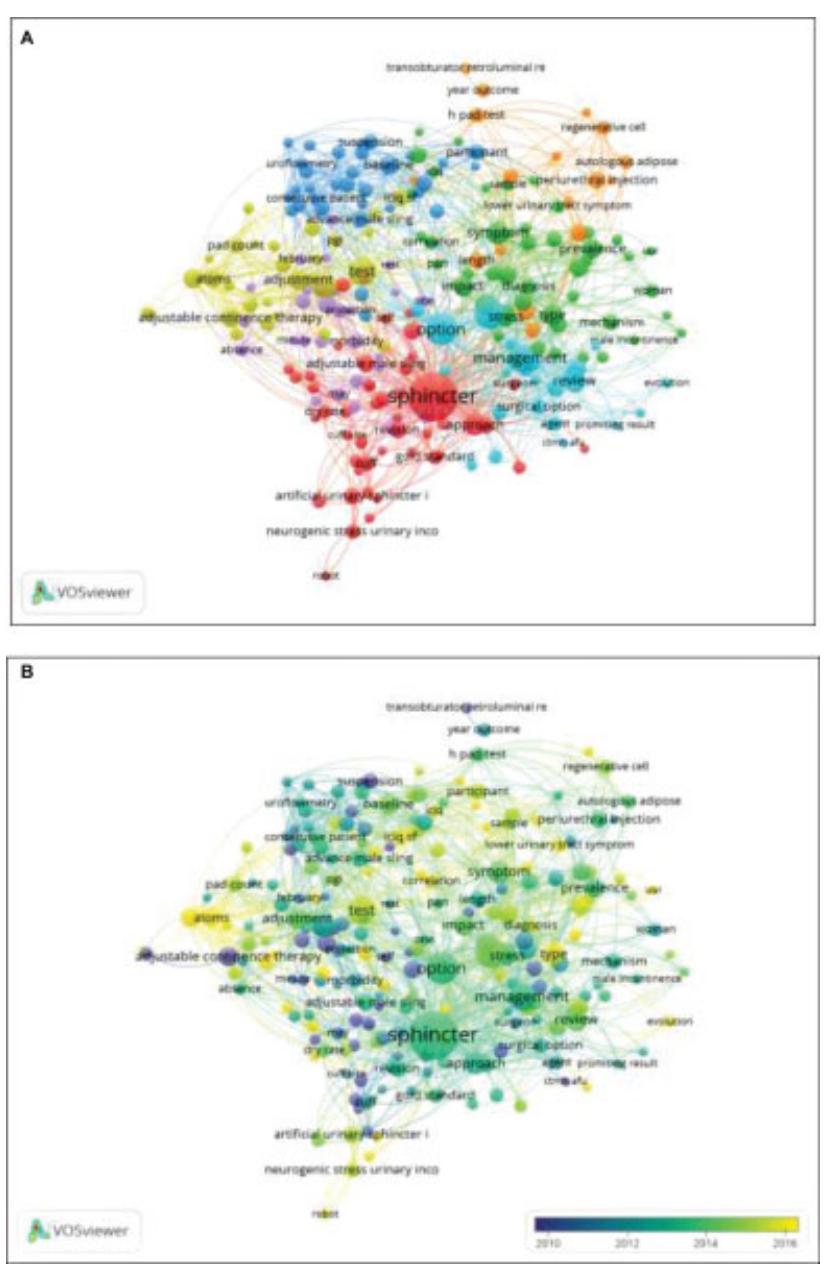

Fig. 4 Análisis de coocurrencia en palabras clave entre título y resumen. (A) Mapeo y agrupamiento de palabras clave en siete grupos. (B) Mapeo y agrupamiento de palabras clave en resúmenes según la frecuencia media de aparición entre 2010 y 2016.

producción intelectual. No hay publicaciones en Colombia con esta estrategia de búsqueda específica, y esto puede estar relacionado con una menor producción intelectual en nuestro país debido al déficit en el reconocimiento económico a la investigación en personal asistencial y menos incentivos y políticas de producción intelectual a nivel gubernamental, comparado con otros países.

En el presente estudio, sólo 5 de las 20 revistas con más publicaciones tienen un FI $\geq 3$. Dentro de los artículos hallados, se encuentra una revisión sistemática que buscaba encontrar las tasas de continencia y complicaciones luego de las cintas en hombres postprostatectomía radical, la cual sólo contaba con estudios observacionales, muy heterogéneos, sin estandarización de terminología, y con altos riesgos de sesgos. ${ }^{17}$ Esto coincide con lo reportado en la sexta consulta internacional en incontinencia publicada en $2018,{ }^{6}$ en que los autores resaltan la necesidad de estudios prospectivos aleatorizados, y con la revisión no sistemática de Kretschmer y Nitti ${ }^{18}$ (2017) en la cual los autores resaltan la falta de estudios aleatorizados que investiguen los desenlaces de las distintas técnicas quirúrgicas para incontinencia urinaria masculina postprostatectomía, y notan que las publicaciones han aumentado, pero que la evidencia es aún insatisfactoria.

Al caracterizar el comportamiento de las publicaciones acerca de incontinencia urinaria masculina en el tiempo mediante análisis por mapeo, encontramos que predominan las publicaciones relacionadas con dispositivos médicos, nuevas tecnologías materiales, y técnicas como la robótica, y se encuentran menos publicaciones acerca de la etiología de la enfermedad. En esto coincidimos con Averbeck et al. ${ }^{6}$ (2018), que afirman la necesidad de contar con estudios clínicos prospectivos y de estandarizar la terminología.

Por último, consideramos que es imperativo crear estudios controlados con términos estandarizados para obtener mejores resultados que puedan publicarse en revistas con mayor FI. Para que esto sea posible, es indispensable el apoyo económico y de colaboración por grupos, para incentivar la investigación en Latinoamérica, lo que permitirá aumentar los datos en nuestra región, generando pautas de manejo acorde a nuestra población, y no adaptando información y conductas de una población muy heterogénea a la nuestra.

\section{Fortalezas}

La evolución año tras año del conocimiento científico conlleva a una gran cantidad de información, lo que hace más difícil su interpretación, especialmente cuando el número de fuentes es muy alto y los conceptos, muy fragmentados. ${ }^{19}$ Es por esto que consideramos que este tipo de estudios es útil para investigar y medir diferentes indicadores, como análisis de conglomerados, tendencias de palabras clave, y otras medidas bibliométricas, pues permite recopilar información a partir de métricas inexploradas que ofrecen información importante para el tema que se está investigando. Nosotros usamos técnicas de análisis de redes para visualizar y detectar tendencias y patrones producidos de forma robusta y replicable en el futuro.

\section{Limitaciones}

La limitación de este estudio consiste en que se realizó la búsqueda en una sola base de datos (WOS), y probablemente se dejó por fuera algunas publicaciones de Latinoamérica y de otras regiones. Adicionalmente, en ocasiones, este tipo de búsqueda sin criterios de exclusión genera fuentes de información que pueden no estar relacionadas del todo con el tema.

\section{Conclusión}

Este es el primer análisis bibliométrico de incontinencia urinaria masculina reportado en la literatura en los últimos veinte años. Se evidencia un patrón de publicación con crecimiento exponencial a lo largo del tiempo; sin embargo, aún existe falta de estandarización en la terminología y déficit de estudios clínicos prospectivos. Latinoamérica cuenta con poca producción científica relacionada con el tema en estudio. 
Consideramos que en nuestro medio se debe incentivar la creación de bases de datos locales que mejoren la producción científica y ayuden a dirigir las pautas de manejo de esta condición.

\section{Conflicto de Intereses}

Los autores no tienen ningún conflicto de intereses que declarar.

\section{Referencias}

1 Abrams P, Andersson KE, Birder L, et al; Members of Committees Fourth International Consultation on Incontinence. Fourth International Consultation on Incontinence Recommendations of the International Scientific Committee: Evaluation and treatment of urinary incontinence, pelvic organ prolapse, and fecal incontinence. Neurourol Urodyn 2010;29(01):213-240

2 Hester AG, Kretschmer A, Badlani G. Male Incontinence: The Etiology or Basis of Treatment. Eur Urol Focus 2017;3(45):377-384

3 Helfand BT, Smith AR, Lai HH, et al; LURN. Prevalence and Characteristics of Urinary Incontinence in a Treatment Seeking Male Prospective Cohort: Results from the LURN Study. J Urol 2018;200(02):397-404

4 Shamliyan TA, Wyman JF, Ping R, Wilt TJ, Kane RL. Male urinary incontinence: prevalence, risk factors, and preventive interventions. Rev Urol 2009;11(03):145-165

5 Bauer RM, Gozzi C, Hübner W, et al. Contemporary management of postprostatectomy incontinence. Eur Urol 2011;59(06): 985-996

6 Averbeck MA, Woodhouse C, Comiter C, et al. Surgical treatment of post-prostatectomy stress urinary incontinence in adult men: Report from the 6th International Consultation on Incontinence. Neurourol Urodyn 2019;38(01):398-406

7 Helfand BT, Smith AR, Lai HH, et al; LURN. Prevalence and Characteristics of Urinary Incontinence in a Treatment Seeking
Male Prospective Cohort: Results from the LURN Study. J Urol 2018;200(02):397-404

8 Cornu J-N, Peyrat L, Haab F. Update in management of male urinary incontinence: injectables, balloons, minimally invasive approaches. Curr Opin Urol 2013;23(06):536-539

9 InCitesTM Journal Citation Reports. Journal data filtered by selected year JCR year: 2014. Thomson Reuters 2015. SCIE, SSCI.

10 Van Eck NJ, Waltman L, Dekker R, Van Den Berg J. A comparison of two techniques for bibliometric mapping: multidimensional scaling and VOS. J Am Soc Inf Sci Technol 2010;61(12):2405-2416

11 Waltman L, van Eck NJ, Noyons ECM. A unified approach to mapping and clustering of bibliometric networks. Journal of Informetrics 2010;4(04):629-635

12 Matta R, Hird AE, Saskin R, et al. Is there an association between urinary incontinence and mortality? A retrospective cohort study. J Urol 2020;203(03):591-597

13 Sahin-Onat S, Unsal-Delialioğlu S, Güzel O, Uçar D. Relationship between urinary incontinence and quality of life/depression in elderly patients. J Gerontol 2014;5(03):86-90

14 Chong JT, Simma-Chiang V. A historical perspective and evolution of the treatment of male urinary incontinence. Neurourol Urodyn 2018;37(03):1169-1175

15 Liu JS, Hofer MD, Milose J, et al. Male sling and artificial urethral sphincter for male stress urinary incontinence among certifying american urologists. Urology 2016;87:95-99

16 Gao Y, Qu B, Shen Y, et al. Bibliometric profile of neurogenic bladder in the literature: a 20-year bibliometric analysis. Neural Regen Res 2015;10(05):797-803

17 Cerruto MA, D'Elia C, Artibani W. Continence and complications rates after male slings as primary surgery for post-prostatectomy incontinence: a systematic review. Arch Ital Urol Androl 2013;85 (02):92-95

18 Kretschmer A, Nitti V. Surgical Treatment of Male Postprostatectomy Incontinence: Current Concepts. Eur Urol Focus 2017;3(4-5):364-376

19 Upham SP, Small H. Emerging research fronts in science and technology: patterns of new knowledge development. Scientometrics 2010;83(01):15-38 